\title{
Evidence of persistent central sensitization in chronic headaches: a multi-method study
}

\author{
Elena Filatova $\cdot$ Nina Latysheva $\cdot$ Alexey Kurenkov
}

Received: 30 May 2008/Accepted: 17 July 2008/Published online: 9 August 2008

(C) Springer-Verlag 2008

\begin{abstract}
The aim of this study was to investigate central sensitization (CS) in chronic headaches and compare this phenomenon between chronic migraine $(\mathrm{CM})$ and chronic tension-type headache (CTTH). We recruited 69 patients with chronic headaches and 18 control subjects. Questionnaires of headache history, allodynia and the Hospital Anxiety and Depression scale were administered. We recorded thresholds for pinprick and pressure pain, blink (BR) and nociceptive flexion reflex (NFR) R3 component coupled with wind-up ratios. Thresholds for pressure and pinprick pain, BR and NFR R3 were lower and wind-up ratios higher in patients. No differences of CS parameters between CM and CTTH were observed. CS is persistent and prevalent in patients with various types of chronic headache. CS levels are unrelated to the predominant side of pain, disease duration or depression. Neither is CS related to the headache type, suggesting similar mechanisms of headache chronification and chronicity maintaining and possibly explaining clinical similarity of various forms of chronic headache.
\end{abstract}

Keywords Allodynia - Chronic headache . Central sensitization · Pressure algometry . Electrophysiology

E. Filatova $\cdot$ N. Latysheva

Sechenov Moscow Medical Academy, Moscow, Russia

E. Filatova $\cdot$ N. Latysheva $(\bowtie) \cdot$ A. Kurenkov

Alexander Vein Headache Clinic, 26/2 Leningradsky pr, 125040 Moscow, Russia

e-mail: ninalat@gmail.com

A. Kurenkov

Research Center for Children's Health, Moscow, Russia

\section{Introduction}

Approximately $3-5 \%$ of the population and up to $70-80 \%$ of patients presenting to headache clinics have daily or near daily headache [1]. The disorder is associated with substantial disability, diminished quality of life, impaired physical, social and occupational functioning [2], and poses a challenging treatment problem.

Clinical experience shows that in course of chronification such pathophysiologically distinct conditions as migraine and tension-type headache (TTH) tend to show increasing clinical similarity. This could be due to a common mechanism contributing to headache chronicity. Two basic mechanisms have been suggested to underlie chronification of different headache types-sensitization of nociceptive structures and deficiency of antinociceptive systems.

Sensitization is the process whereby the stimulus needed to generate a response decreases over time, while the amplitude of the response to any given stimulus increases [3]. In migraine, central sensitization (CS) is described in the spinal trigeminal nucleus, which receives convergent input from the dura and the face $[4,5]$. Pain arising from innocuous stimulation of the skin is known as allodynia, which is currently considered a clinical correlate of CS [4, $6,7]$. In fact, many patients report sensitivity to shaving, hair brushing, wearing glasses and jewelry during a migraine attack. In TTH, several lines of evidence indicate that the development of sensitization at the level of the spinal dorsal horn/trigeminal nucleus is the result of prolonged continuous painful input from pericranial myofascial tissues [8]. In episodic migraine (EM) and episodic tension-type headache CS has been thoroughly studied [4, 7, 9], while the evidence of CS in chronic headaches is still scarce $[8,10-12]$. 
Various methods have been employed to study CSquantitative sensory testing to assess heat and cold pain thresholds [7], von Frey filaments [10, 12] and pressure pain thresholds (PPT) [13] for mechanical allodynia. Electrically elicited blink (BR) and nociceptive flexion reflexes (NFR) have been extensively used to assess pain sensitivity [14, 15]. The BR R3 component is thought to follow a medullar pathway involving trigeminal circuits. The NFR R3 threshold reduction has been accepted as evidence of antinociceptive deficiency. There are certain difficulties in interpreting the results obtained, as pain sensitivity is assessed by various techniques which diagnostic value has not been compared.

The objective of this study was to investigate CS in chronic headache with a variety of methods and compare this phenomenon across chronic migraine (CM) and chronic tension-type headache (CTTH).

\section{Methods}

The study was approved by the local ethics committee of Sechenov Moscow Medical Academy and was performed in accordance with the ethical standards of the 1964 Declaration of Helsinki. All participants signed a document of informed consent prior to their inclusion in the study.

A total of 69 patients with IHS-defined CM, CTTH [16] or mixed chronic headache were recruited from the Alexander Vein Headache Clinic or were referred from municipal outpatient clinics. Control subjects were relatives of patients with no appreciable headache problem recruited from the clinic's waiting room. Control subjects were used rather than normative data because it was unlikely that the conditions of the normative trials could be exactly reproduced, and it has been suggested that normative data in pain threshold testing may not be reliable in different study populations [17].

Exclusion criteria for all groups were age under 18 or over 65 , the presence of peripheral neuropathy, dermatological disease, chronic pain in another location, major psychiatric disorder. All participants were not allowed to be on headache-preventive medications (all preventives had to be discontinued at least 3 months prior to the study) or take rescue medications within $6 \mathrm{~h}$ before the examination. All patients were studied during their mildest headaches (the pain intensity range was $0-5 \mathrm{~cm}$ on the 10$\mathrm{cm}$ visual analogue scale (VAS), with the mean intensity of $2.3 \mathrm{~cm}$ ).

A questionnaire of demographic data, headache history and clinical features was administered. In addition, an allodynia questionnaire proposed by LoPinto et al. [12] and the Hospital Anxiety and Depression scale (HADS) were administered.
All assessments were carried out by one investigator.

Pressure allodynia (PA) was tested by using a pressure algometer in five bilateral skin areas on the head and neck (forehead V1, temple, parietal area, posterior neck/C2, shoulder/trapezius) (Fig. 1). PPT is defined as the amount of pressure required to change a sensation of pressure to a sensation of pressure and pain [18]. A hand-held pressure algometer (Commander Algometer ${ }^{\mathrm{TM}}$, JTech) was used. The device displays values in $\mathrm{lb} / \mathrm{cm}^{2}$, ranging from 0 to 20 $\mathrm{lb}$, with $0.1 \mathrm{lb}$ intervals. PA was also tested by using a mechanical pinprick stimulator (Neuropen ${ }^{\circledR}$, Owen Mumford) which allows exerting a quantifiable force of $40 \mathrm{~g}$ in the same skin areas. The degree of pain or unpleasant sensation upon stimulation was measured using a $100-\mathrm{mm}$ VAS. For both tests, two consecutive measurements in each point with intervals of $20 \mathrm{~s}$ were obtained. The mean score was used for further analysis.

The wind-up phenomenon (temporal summation of pain) was induced by a pinprick followed by a train of 10 pinpricks applied repeatedly at $1 \mathrm{~Hz}$ on a skin area of $1 \mathrm{~cm}^{2}$ on the forehead, temple and neck with the Neuropen ${ }^{\circledR}$ stimulator. All participants assessed the intensity of pain induced by a single pinprick and the whole train of stimuli using the 100-mm VAS scale. Wind-up ratio was calculated as VAS (train of stimuli)/VAS (initial single pinprick).

An algometry score was determined as the sum of pressure pain thresholds at all sites. Pinprick and wind-up scores were calculated similarly.

Forty patients with chronic headache and all control subjects underwent BR and NFR testing.

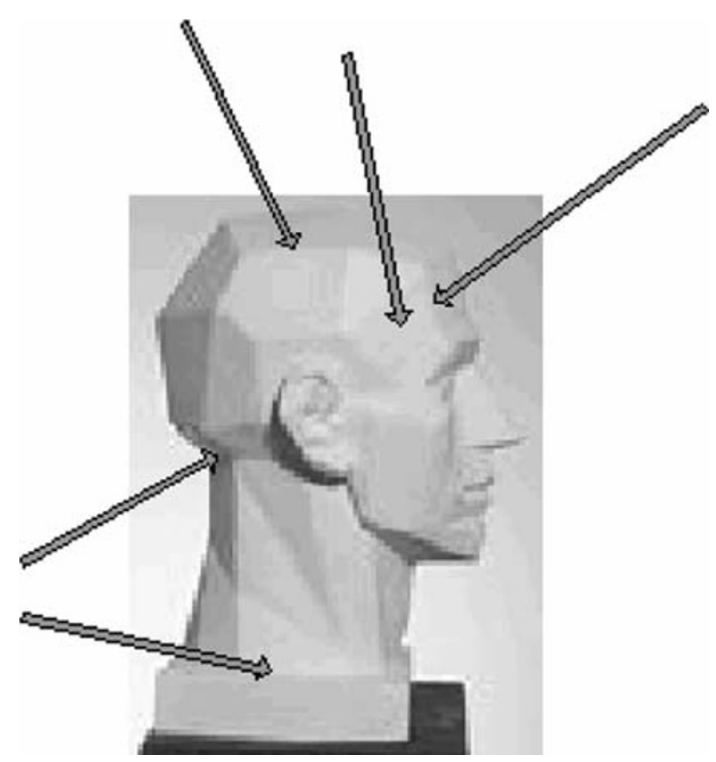

Fig. 1 Skin areas of pressure algometry, pinprick and wind-up testing 
BR: Electromyography (EMG) signals were recorded on the right side from the orbicularis oculi muscles with a surface electrode attached to the outer-lower eyelid. Reference electrode was attached on the nose dorsum and a ground electrode was attached to the wrist. BR was elicited by electrical stimulation of the right supraorbital nerve delivered via 5-mm diameter surface electrodes. The cathode was placed over the supraorbital foramen and the anode was placed approximately $1 \mathrm{~cm}$ rostrally on the forehead. The stimulation consisted of $0.2 \mathrm{~ms}$ rectangular single pulses delivered with arbitrary intervals of not less than $30 \mathrm{~s}$ to minimize habituation. EMG signals were amplified by biopotential amplifiers (Keypoint ${ }^{\circledR}$ Portable, Medtronic) with the bandwidth of $10 \mathrm{~Hz}-5 \mathrm{kHz}$. We analyzed the thresholds of the ultralate R3 component, probably following a multisynaptic trigeminal pathway in the medullar reticular formation involving antinociceptive structures such as periaqueductal gray and raphe nuclei [19]. Ellrich et al. [20] showed that cutaneous A-beta and A-delta fibres constitute the generators of the electrically elicited R3 component.

NFR: The surface stimulating electrodes were placed at a retromalleolar location along the course of the right sural nerve, $2 \mathrm{~cm}$ apart, with the cathode placed proximally. The recording surface electrodes were placed on the tendon (reference electrode) and over the belly of the biceps femoris capitis brevis muscle (active electrode). The stimulus consisted of pulse trains of $20 \mathrm{~ms}$ duration, with an interval frequency of $300 \mathrm{~Hz}$ and arbitrary intervals of not less than $15 \mathrm{~s}$. EMG signals were amplified by biopotential amplifiers (Keypoint ${ }^{\circledR}$ Portable, Medtronic) with the bandwidth of $20 \mathrm{~Hz}-5 \mathrm{kHz}$. We registered the R3 (reflex) threshold and the subjective pain threshold.

Data were analyzed using Statistica software (MannWhitney $U$ test for between-group comparisons, Wilcoxon test to assess side-to-side differences, Spearman correlations, Fisher's exact test to compare proportions). All values are shown as mean \pm standard deviation (SD).

\section{Results}

Patient demographics and clinical features of chronic headache

The headache group included 25 patients with CM, 25 patients with CTTH and 19 patients with mixed chronic headache (CM or CTTH plus cervicogenic headache), according to ICHD-2 [16]. Mean headache frequency was 25 days per month with the mean pain intensity of $7 \mathrm{~cm}$. With an average 18-year headache history, our patients reported having chronic headache for 6 years. Analgesic overuse was present in $20(29 \%)$ patients. When filling the allodynia questionnaire, patients were asked to give a positive response if allodynia was present at any momentduring the baseline headache, its exacerbations or both. Based on the questionnaire, the prevalence of cutaneous allodynia was $79 \%$. Our patients reported low depression (7 points) and mild anxiety levels (10 points) (Table 1$)$.

Pain sensitivity in chronic headache

Pressure pain sensitivity was consistently higher among headache patients than among controls across all sites except temples, pinprick pain and wind-up ratios-across all sites except the forehead. In the CM subgroup, no differences in any measured parameters were observed between the predominant side of pain and the contralateral side.

In the headache group significant reduction of the algometry and increase of the pinprick scores were observed ( $P=0.004$ and $P=0.0008$, respectively), compared with controls. In the headache group, we also found significant reduction of the BR R3 threshold (7.6 \pm 2.16 vs. $9.6 \pm 1.5 \mathrm{~mA})$, NFR R3 threshold $(9.0 \pm 2.2$ vs. $10.3 \pm 1.7$ $\mathrm{mA})$ and NFR pain threshold $(6.2 \pm 1.9$ vs. $9.8 \pm 1.8 \mathrm{~mA})$ (Table 2).

As there are no standard cut-off values for allodynia in algometry, we used the control group to define PA. To classify a subject's response as allodynic, the cut-off value for allodynia was defined as the algometry score level, above which $95 \%$ of control subjects responded. This cutoff value was $41.9 \mathrm{lb}$. Based on this score, the prevalence of pressure allodynia in the headache group was $70 \%$. Based on the pinprick score, allodynia prevalence was $58 \%$, while based on both algometry and pinprick scores, $79.7 \%$.

To compare the diagnostic value of the simple pressure algometry technique coupled with a wind-up assessment

Table 1 Patient demographics and clinical features of chronic headache

\begin{tabular}{lll}
\hline & Headache & Control \\
\hline Group size & 69 & 18 \\
Gender (female:male) & $57: 12$ & $13: 5$ \\
Age (years) & $40.1 \pm 12.3$ & $43.1 \pm 18.1$ \\
Headache frequency (days/month) & $25.2 \pm 6.0$ & \\
Pain intensity (VAS) & $7.1 \pm 1.9$ & \\
Headache history (years) & $18.2 \pm 12.7$ & \\
Chronic headache history (years) & $5.7 \pm 7.0$ & \\
Cutaneous allodynia & $79 \%$ & \\
Depression & $7.4 \pm 3.5$ & \\
Anxiety & $9.7 \pm 3.9$ & \\
Pain intensity at exam (VAS) & $2.3 \pm 1.9$ & 0 \\
\hline
\end{tabular}


Table 2 Pain sensitivity in chronic headache

\begin{tabular}{lccl}
\hline & Headache & Control & $\begin{array}{l}\text { Group } \\
\text { differences }\end{array}$ \\
\hline Algometry score & $40.64 \pm 13.4$ & $52.5 \pm 16.7$ & $P<0.005^{*}$ \\
Pinprick score & $54.76 \pm 62.9$ & $19.08 \pm 21.6$ & $P<0.005^{*}$ \\
Wind-up score & $24.07 \pm 24.37$ & $12.4 \pm 8.3$ & $\mathrm{NS}$ \\
BR R3 (mA) & $7.6 \pm 2.16$ & $9.6 \pm 1.5$ & $P<0.0001^{*}$ \\
NFR R3 (mA) & $9.0 \pm 2.2$ & $10.3 \pm 1.7$ & $P<0.005^{*}$ \\
$\begin{array}{l}\text { NFR pain threshold } \\
\text { (mA) }\end{array}$ & $6.2 \pm 1.9$ & $9.8 \pm 1.8$ & $P<0.0001^{*}$ \\
\hline
\end{tabular}

* Significant differences

$N S$ not significant

and a more sophisticated and time-consuming BR or NFR testing, we defined the cut-off values for BR and NFR R3 thresholds as described above. These cut-off values were 8.9 $\mathrm{mA}$ for the BR R3 and $9.45 \mathrm{~mA}$ for the NFR R3 threshold. Based on these cut-off values, the prevalence of CS was $76 \%$ as defined by BR and $62 \%$ by NFR. The diagnostic sensitivity of the algometry + wind-up combination (allodynia was considered present if at least one method yielded a positive result) differed neither from BR nor from NFR (McNemar's test, $P=1.0$ ).

No correlations between such clinical characteristics as headache frequency, chronic or total headache history and pain sensitivity parameters were observed.

Comparison of clinical headache characteristics and allodynia between CM and CTTH

For comparison across headache types, 25 patients with CM and 25 patients with CTTH were enrolled. Both headache groups were comparable from the demographical and clinical perspective. Patients with CM (68\%) and with CTTH $(64 \%)$ reported symptoms of cutaneous allodynia (Table 3).

No significant difference in any of the pain sensitivity parameters was observed between CM and CTTH (Fig. 2).

The prevalence of PA was $60 \%$ in the CM group and $68 \%$ in the CTTH group (no between-group differences, Fisher's exact test, $P=0.7$ ).

\section{Discussion}

This study presents a comprehensive analysis of CS in patients with chronic headaches during their mildest pain and uses various methods-pressure algometry, pinprick and wind-up assessments and more sophisticated neurophysiologic techniques. In addition, the study features a comparative analysis of CS across different types of chronic headache.
Table 3 Patient demographics and clinical features of $\mathrm{CM}$ and CTTH

\begin{tabular}{lll}
\hline & CM & CTTH \\
\hline Group size & 25 & 25 \\
Gender (female:male) & $23: 2$ & $23: 2$ \\
Age (years) & $44.5 \pm 12.6$ & $39.5 \pm 10.0$ \\
Headache frequency (days/month) & $23.5 \pm 6.2$ & $25.1 \pm 6.0$ \\
Pain intensity (VAS)* & $8.7 \pm 1.4$ & $6.2 \pm 1.6$ \\
Chronic headache history (years) & $6.6 \pm 7.5$ & $4.8 \pm 6.0$ \\
Cutaneous allodynia (\%) & 68 & 64 \\
Depression & $6.5 \pm 4.0$ & $7.6 \pm 3.2$ \\
Anxiety & $9.5 \pm 4.7$ & $10.9 \pm 3.5$ \\
Pain intensity at exam (VAS) & $2.44 \pm 2.3$ & $2.52 \pm 1.9$ \\
\hline
\end{tabular}

* Significant difference

Our study has shown that patients with chronic headache exhibit both pressure and pinprick allodynia, and increased temporal summation of pain. Our data is in keeping with other authors reporting reduced pressure pain thresholds as evidence of CS $[13,21]$. Wind-up has been suggested to be responsible for the induction and maintenance of central sensitization. Although it is not equivalent to $\mathrm{CS}$, it is a progressive, frequency-dependent increase in C-fibre evoked responses of trigeminal and spinal dorsal WDR neurons [22].

In chronic headaches, CS can be stably detected during the baseline headache and even in the headache-free period. In our study, allodynia prevalence was $79 \%$ based on the allodynia questionnaire, $70 \%$ based on the pressure pain testing and $79.7 \%$ based on pressure and pinprick pain testing. Cooke et al. [10] who assessed pain sensitivity with von Frey filaments reported a $75 \%$ prevalence. Our data is also in keeping with Burstein et al. [4] who defined allodynia thresholds in mechanical, heat and cold sensory tests and achieved a higher sensitivity for detection of allodynia by testing three allodynia modalities.

Burstein et al. [7] showed that during the migraine attack cutaneous allodynia develops on the pain side and spreads to the contralateral side only in prolonged attacks. At the same time, our study has demonstrated that patients with chronic headaches exhibit bilateral CS, which is unrelated to the predominant side of pain during exacerbations. Absence of side-to-side differences was also reported in CTTH [13]. These findings suggest that CS is stable and permanent in chronic headaches. Yet, in EM, allodynia has been shown to be less persistent and less prominent and temporarily related to migraine attacks.

Having evolved to a daily or near-daily pattern, pain becomes independent from specific triggers and is sustained by the stable CS of nociceptive structures.

As there are no correlations between CS and the length of headache history, factors other than duration might 
Fig. 2 Non-significant differences in algometry, pinprick and wind-up scores, BR R3, NFR R3 and NFR pain thresholds between CM (striped bars) and CTTH (full bars)

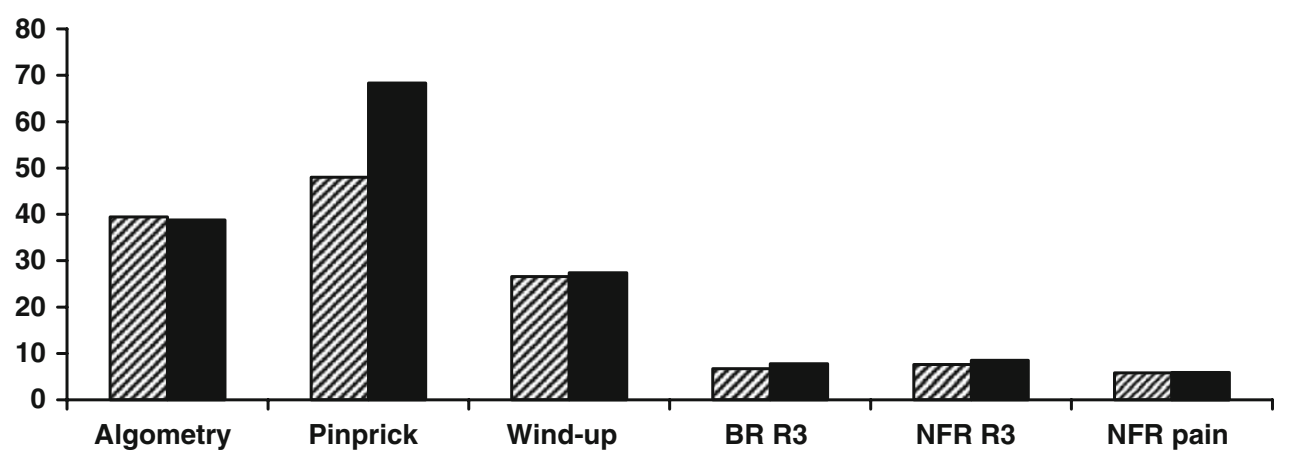

contribute to its chronification. Clinical evidence shows that some patients report having episodic headaches for years, others, vice versa, rather quickly progress towards frequent and transformed headache patterns. This means that clinicians need to examine headache patients thoroughly to detect the first signs of CS even in patients with short headache history. The presence of allodynia may support decisions to undertake early and aggressive preventive treatment in patients with episodic headache presenting with CS in the headache-free period.

Anxiety and depression have been suggested to contribute to headache chronification and share similar neurobiological mechanisms [23]. Our patients reported subclinical levels of depression and anxiety.

Anecdotal evidence shows that triptan efficacy is markedly reduced in CM. Burstein et al. [24] provided convincing evidence for the 2-h time window for triptans, i.e. they should be administered during the period of peripheral sensitization, before cutaneous allodynia develops. This may be, however, a challenge for patients with chronic headaches, as very often pain is continuous and cutaneous allodynia is detected already during the mild headache and even in the pain-free period. In this light, CS appears to underlie intractable headaches.

This study has also shown no differences in allodynia levels between CM and CTTT. These data are in line with the clinical evidence showing that once the process of chronification is underway, such clinically distinct disorders as migraine and tension-type headache tend to show increasing clinical similarity. This means that persistent CS, detected during baseline headache, may reflect a common pathological mechanism of headache chronification and chronicity maintaining.

The reduced BR and NFR R3 thresholds we observed are in keeping with other studies [14]. The R3 component has been shown to be nociceptive in origin [25], as it appears after low intensity and potentially not dangerous stimuli and is markedly reduced by anesthetic block. R3 could be interpreted as an expression of a possible primary dysfunction of the trigeminal reflex circuits probably caused by a failure of central control on the brainstem neuronal networks [26]. The incoming nociceptive impulses converging on the trigeminal nucleus caudalis result in pain summation (wind-up), a decrease in activation threshold (CS), a parallel decrease in headache attack threshold and, finally, headache chronification [25].

However, very recently Ellrich et al. [20] showed that cutaneous A-beta and A-delta, but not C-fibres constitute the generators of the electrically elicited R3 component. One of the limitations of this study is the recording of BR by using the standard stimulus electrode. The 'nociceptive' $\mathrm{BR}$ (nBR) is possible to elicit by a custom-built planar concentric electrode providing a high current density at low intensities via a central cathode of $1 \mathrm{~mm}^{2}$ [27]. While some authors note that R3 abnormalities detected in migraine may be ascribed to a general dysfunction of adaptation capacity to environmental conditions [28], nBR may be a more suitable method for CS research once the electrodes become commercially available.

While BR testing yields results similar to other techniques, and A-delta fibres are involved in the mediation of the R3 component, reduced R3 thresholds probably reflect $\mathrm{CS}$ in the trigeminal circuits and may be a reliable measure of CS.

NFRs are related to A-delta fibre activation. Reduced NFR thresholds have led many authors to conclude that chronic headache is a disorder of endogenous antinociceptive systems [15, 29]. Impairment of endogenous supraspinal pain modulation systems opens up the way for CS in the spinal trigeminal nucleus.

In our study, allodynia was assessed using a variety of techniques. Although there is uncertainty about the diagnostic sensitivity of these methods, we showed that simultaneous usage of a whole battery of techniques might improve their sensitivity in the detection of CS. Our study has shown similar prevalence of allodynia as detected by each method. Moreover, our results are in line with the previous reports on CS in headaches [4, 10, 14, 21, 29]. As these methods give comparable results, in the tight periods of a consultation neurologists can use such simple and time efficient methods as pressure algometry and wind-up testing to detect the presence of CS. 
Future studies of CS in chronic headaches are required, as deeper understanding of this phenomenon may shed light on the mechanisms of headache chronification, causes of its intractability, as well as provide a firm basis for novel pathogenesis-based methods of treatment.

This comprehensive study shows that most patients with chronic headache exhibit CS during baseline headache detectable by various techniques. CS level does not depend on such clinical headache characteristics as frequency, duration or predominant side of pain. Neither are CS prevalence and level related to the headache form, suggesting that CS may be a universal mechanism of headache chronification and chronicity maintaining.

Acknowledgments The authors wish to express their sincere thanks to $\mathrm{V}$. Oknin, $\mathrm{PhD}$ for the useful discussions, and to the patients and control subjects who volunteered their time to participate in this research. Nina Latysheva was supported by a doctoral research scholarship from Moscow Medical Academy.

Conflict of interest None.

\section{References}

1. Dodick D (2006) Chronic daily headache. N Eng J Med 354:158165

2. D'Amico D, Usai S, Grazzi L et al (2003) Quality of life and disability in primary chronic daily headache. Neurol Sci 24(Suppl 2):97-100

3. Ossipov MH, Lai J, Malan TP Jr, Porreca F (2000) Spinal and supraspinal mechanisms of neuropathic pain. Ann NY Acad Sci 909:12-24

4. Burstein R, Yarnitsky D (2000) An association between migraine and cutaneous allodynia. Ann Neurol 47(5):614-624

5. Burstein R (2001) Deconstructing migraine headache into peripheral and central sensitization. Pain 89:107-110

6. Woolf CJ (1983) Evidence for a central component of post-injury pain hypersensitivity. Nature 306:686-688

7. Burstein R, Cutrer M, Yarnitsky D (2000) The development of cutaneous allodynia during a migraine attack. Clinical evidence for the sequential recruitment of spinal and supraspinal nociceptive neurons in migraine. Brain 123:1703-1709

8. Bendtsen L (2003) Central and peripheral sensitization in tensiontype headache. Curr Pain Headache Rep 7(6):460-465

9. Mørk H, Ashina M, Bendtsen L, Olesen J, Jensen R (2004) Possible mechanisms of pain perception in patients with episodic tension-type headache-a new experimental model of myofascial pain. Cephalalgia 24(6):466-75

10. Cooke L, Eliasziw M, Becker WJ (2007) Cutaneous allodynia in transformed migraine patients. Headache 47(4):531-539
11. Kitaj M, Klink M (2005) Pain thresholds in daily transformed migraine versus episodic migraine headache patients. Headache 45:992-998

12. LoPinto C, Young WB, Ashkenazi A (2006) Comparison of dynamic (brush) and static (pressure) mechanical allodynia in migraine. Cephalalgia 26:852-856

13. Fernández-de-las-Peñas $\mathrm{C}, \mathrm{Ge} \mathrm{HY}$, Cuadrado ML, Madeleine $\mathrm{P}$, Pareja JA, Arendt-Nielsen L (2008) Bilateral pressure pain sensitivity mapping of the temporalis muscle in chronic tension-type headache. Headache 48:1067-1075

14. De Tommaso M, Sciruiccio V, Puca A (2000) Clinical neurophysiology of chronic daily headache. J Headache Pain 1:S81-S87

15. Sandrini G, Rossi P, Milanov I, Serrao M, Cecchini AP, Nappi G (2006) Abnormal modulatory influence of diffuse noxious inhibitory controls in migraine and chronic tension-type headache patients. Cephalalgia 26:782-789

16. Headache Classification Committee of the International Headache Society (2004) The International Classification of Headache Disorders. Cephalalgia 24(Suppl 1):1-160

17. Yarnitsky D (1997) Quantitative sensory testing. Muscle Nerve 20:198-204

18. Fischer AA (1990) Application of pressure algometry in manual medicine. J Man Med 5:145-150

19. Ellrich J, Hopf HC (1996) The R3 component of the blink reflex: normative data and application in spinal lesions. Electroencephogr Clin Neurophysiol 101:349-354

20. Ellrich J, Katsarava Z, Przywara S, Kaube H (2001) Is the R3 component of the human blink reflex nociceptive in origin? Pain 91:389-395

21. Ashina S, Babenko L, Jensen R, Ashina M, Magerl W, Bendtsen L (2005) Increased muscular and cutaneous pain sensitivity in cephalic region in patients with chronic tension-type headache. Eur J Neurol 12:543-549

22. Coste J, Voisin DL, Luccarini P, Dallel R (2008) A role for windup in trigeminal sensory processing: intensity coding of nociceptive stimuli in the rat. Cephalalgia 28(6):631-639

23. Hamelsky S, Lipton R (2006) Psychiatric comorbidity of migraine. Headache 46:1327-1333

24. Burstein R, Collins B, Jakubowski M (2004) Defeating migraine pain with triptans: a race against the development of cutaneous allodynia. Ann Neurol 55:19-26

25. Proietti Cecchini A, Sandrini G, Fokin IV, Moglia A, Nappi G (2003) Trigeminofacial reflexes in primary headaches. Cephalalgia 23(Suppl 1):33-41

26. De Tommaso M, Guido M, Libro G, Sciruicchio V, Losito L, Difruscolo O, Puca A (2000) The blink reflex in chronic cluster headache: a comparison with migraine patients suffering from unilateral pain. J Headache Pain 2:97-104

27. Kaube H, Katsarava Z, Kaufer T, Diener HC, Ellrich J (2000) A new method to increase nociception specificity of the human blink reflex. Clin Neurophysiol 111:413-416

28. De Tommaso M (2008) Author's reply. Cephalalgia 28(6):677678

29. Langemark M, Bach FW, Jensen TS, Olesen J (1993) Decreased nociceptive flexion reflex threshold in chronic tension-type headache. Arch Neurol 50:1061-1064 